\title{
Self-management in bronchiectasis: the patients' perspective
}

\author{
K. Lavery*,\#, B. O’Neill ${ }^{\#}$, J.S. Elborn*, ${ }^{\star}$, J. Reilly ${ }^{+}$and J.M. Bradley*,\#
}

ABSTRACT: Self-management programmes for chronic disease are a high priority for healthcare providers. The content and method of delivery of self-management should give consideration to the specific requirements of the disease population. The aims of the present study were to assess the physical and psychosocial impact of bronchiectasis, to determine whether patients with bronchiectasis are receptive to self-management and to identify any obstacles or sources of support for a disease-specific self-management programme.

A total of $\mathbf{3 2}$ patients with a diagnosis of bronchiectasis attended four focus groups. Each focus group was videotaped and subjected to qualitative analysis using the grounded theory approach.

Bronchiectasis had an impact on patients' physical and psychosocial well-being. Patients demonstrated the potential to self-manage with strategies including self-regulation of medication and airway clearance. Perceived obstacles to self-management included lack of information and confidence. Patients suggested that self-management could be promoted through diseasespecific information and appropriate healthcare procedures.

In summary, patients with bronchiectasis have their lives disrupted by this chronic condition, but are receptive to self-management. The present study has provided information from the patients' perspective of elements which need to be included in a successful disease-specific selfmanagement programme.

KEYWORDS: Bronchiectasis, focus groups, self-management

B ronchiectasis is a chronic lung condition characterised by damage to the bronchial wall causing abnormal airway dilatation, poor mucus clearance and pooling of mucus in the affected areas [1]. Symptoms include chronic cough, mucus production, dyspnoea, haemoptysis, malaise, weight loss and repeated exacerbations [2]. As this is a long-term condition, patients have to cope with these symptoms over the course of their life. Patients with bronchiectasis may have a reduced quality of life and increased anxiety and depression $[3,4]$.

Current management of bronchiectasis includes antibiotic therapy, airway clearance, exercise, pulmonary rehabilitation and a range of inhaled medication [2]. The World Health Organization (WHO) advocates the addition of self-management programmes for long-term conditions, such as bronchiectasis, to enhance patient care [5]. Selfmanagement refers to the way in which patients manage their day-to-day care over the length of their disease and includes adherence to medical treatments, actions to prevent ill health, promotion of good health, dealing with psychological and social needs, and communication with healthcare practitioners $[6,7]$.
Self-management programmes offer the potential to include disease-specific self-management strategies such as those available for asthma, chronic obstructive pulmonary disease (COPD) and cystic fibrosis (CF) [8-10]. There are no disease-specific self-management strategies for bronchiectasis and the development of such a programme needs to ensure that the specific needs of the disease population are identified and included [11]. The present focus group study was conducted to explore the concept of self-management in depth, from the patients' perspective. Focus groups have been used previously to assess the needs of patients and for developing new or redefining services [12]. They have a high level of face validity because elements of the discussion can be immediately confirmed or disputed among the participants.

The specific aims of the present study were to: investigate the impact of bronchiectasis on patients' physical and psychosocial functioning; identify whether patients with bronchiectasis were engaging in self-management; identify any perceived obstacles to self-management; and identify perceived sources of support for selfmanagement.

\section{AFFILIATIONS}

*Dept of Respiratory Medicine, Belfast City Hospital,

'Dept of Respiratory Medicine, Queen's University Belfast, Belfast, "Health and Rehabilitation Sciences Research Institute, and +UNESCO Centre, University of Ulster, Newtownabbey, Northern Ireland, UK.

\section{CORRESPONDENCE}

J.M. Bradley

Room 14J02

Health and Rehabilitation Sciences

Research Institute

School of Health Sciences University of Ulster

Shore Road

Newtownabbey

Northern Ireland

BT37 OQB

UK

Fax: 442890368419

E-mail: jm.bradley@ulster.ac.uk

Received:

April 282006

Accepted after revision:

October 062006

\section{SUPPORT STATEMENT}

This study was funded by the Research \& Development Office

Belfast, Northern Ireland, UK.

STATEMENT OF INTEREST

None declared. 


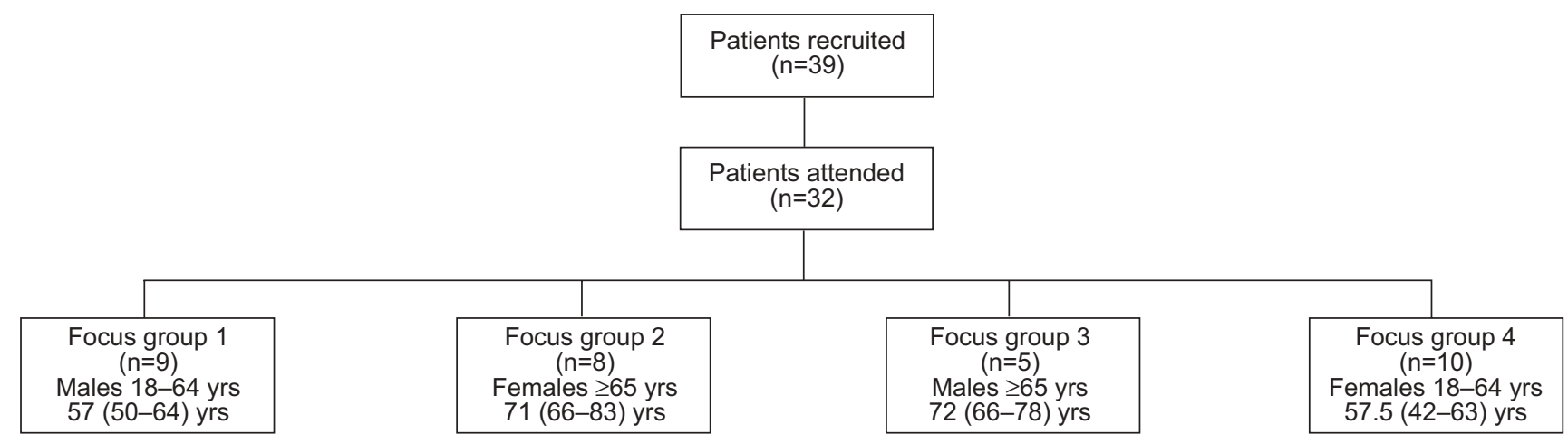

FIGURE 1. Focus group recruitment and profiles. Data are expressed as median (range).

\section{METHODS}

\section{Ethics}

The local medical research ethics committee approved the study and the patients gave informed written consent.

\section{Recruitment}

A total of 39 patients with bronchiectasis who regularly attend specialist respiratory clinics at Belfast City Hospital were recruited. They attended four focus groups, which is the number often recommended to obtain theoretical saturation for homogenous groups. The focus groups were segmented according to sex and age (fig. 1). Inclusion criteria were: close proximity to the hospital; confirmation of diagnosis by computed tomography; and the presence of airway obstruction. Exclusion criteria were: oxygen dependency; colonisation of Pseudomonas aeruginos or methicillin-resistant Staphlococcus aureus in sputum tests; communication difficulties; mobility problems; participation in a previous trial within 3 months; and a history of nonattendance. Characteristics of patients attending the focus groups are outlined in table 1.

\section{Data collection}

An experienced moderator facilitated the focus groups. The

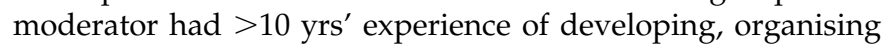
and facilitating focus groups on a range of topics, and coding and analysing the resulting data. A structured schedule of topics was developed, which was used to guide the discussion. The questions were reviewed by the moderator prior to the focus groups and modified to ensure the questions were openended (table 2). All four focus groups were videotaped.

\section{Analysis}

Analysis of the focus groups was conducted in four stages: debriefing, transcription, selective coding, and triangulation. Saturation of subcategories was obtained by the fourth focus group.

\section{Debriefing}

A debrief was conducted the day after each focus group with the specialist physiotherapist and specialist nurse. Group norms and conflicting views were identified for each focus group. Topics discussed in each focus group were reviewed and compared to the previous group. The schedule of topics for the subsequent focus group was modified as appropriate, ensuring that any important additional issues were explored.

\section{Transcription}

The investigator manually transcribed the recordings of each focus group.

\section{Selective coding}

Coding was based on the grounded theory approach to qualitative analysis. Quotes relating to one topic were grouped together as subcategories and integrated under four main core categories, which related to the aims of the study. The four main core categories were as follows: 1) physical and psychosocial impact of bronchiectasis; 2) self-management strategies used by patients with bronchiectasis; 3) perceived obstacles to self-management of bronchiectasis; and 4) perceived sources of support for self-management of bronchiectasis.

\section{Triangulation}

A second researcher verified the core and subcategories. Member checking and a follow-up questionnaire supported the results of the analysis.

\begin{tabular}{|c|c|c|c|c|}
\hline & Group 1 & Group 2 & Group 3 & Group 4 \\
\hline Subjects $n$ & 9 & 8 & 5 & 10 \\
\hline Sex & Male & Female & Male & Female \\
\hline Age yrs & $18-64$ & $\geqslant 65$ & $\geqslant 65$ & $18-64$ \\
\hline \multicolumn{5}{|l|}{$\begin{array}{l}\text { Lung function } \\
\text { category } \mathbf{n}^{\#}\end{array}$} \\
\hline Mild & 0 & 0 & 0 & 0 \\
\hline Moderate & 4 & 2 & 3 & 8 \\
\hline Severe & 5 & 5 & 1 & 1 \\
\hline Very severe & 0 & 1 & 1 & 1 \\
\hline $\begin{array}{l}\text { Hospital } \\
\text { admissions } \mathbf{n}\end{array}$ & 1 & 3 & 1 & 1 \\
\hline $\begin{array}{l}\text { Respiratory } \\
\text { clinic visits }\end{array}$ & $1(0-3)$ & $1(0-1)$ & $2(0-6)$ & $1(0-8)$ \\
\hline $\begin{array}{c}\text { Antibiotic } \\
\text { courses }\end{array}$ & $4(3-4)$ & $1.5(1-3)$ & $4(0-6)$ & $4(0-8)$ \\
\hline $\begin{array}{l}\text { Data are presented } \\
\text { Global Initiative for } \\
\text { the previous } 6 \text { mor }\end{array}$ & $\begin{array}{l}\text { median (ra } \\
\text { onic Obstr }\end{array}$ & $\begin{array}{l}\text {, unless oth } \\
\text { e Lung Dis }\end{array}$ & $\begin{array}{l}\text { se stated. } \\
\text { guidelines }\end{array}$ & $\begin{array}{l}\text { cording to } \\
\text { ?: within }\end{array}$ \\
\hline
\end{tabular}




\section{TABLE 2 Schedule of topics for focus group study}

1. What does self-management mean to you?

2. What aspects of your bronchiectasis are currently easy to self-manage?

3. What aspects of your bronchiectasis are currently difficult to self-manage?

4. How could self-management be improved in relation to information about medication, physiotherapy, nutrition, exercise and hospital admissions?

5. What contact do you currently have with the bronchiectasis service?

What is good about that contact?

What is not so good about that contact?

6. How could the bronchiectasis service help you manage your bronchiectasis better at home?

7. Are there other measures that could help you manage your bronchiectasis better at home e.g. childcare, care of elderly relatives, assistance from other agencies?

8. Why would you like to be able to self-manage?

9. What sorts of issues limit self-management generally?

\section{RESULTS}

\section{Core category 1: effect of bronchiectasis on physical and psychosocial functioning}

Patients reported that bronchiectasis had an impact on their physical and psychosocial functioning (table 3).

Reduced ability to do house work and leisure activities were consistent subcategories discussed in all four focus groups in relation to the physical impact of bronchiectasis, illustrated by the following quotes. "I find if I am vacuuming just maybe a couple of square meters I would be out of puff and have to sit down...ten years ago I was able to build my own house" (male, 18-64 yrs); and "I play bowls but I've had to give up" (male, $\geqslant 65$ yrs).

Emotional issues related to having bronchiectasis, which were frequently due to symptoms, were consistently discussed in all four focus groups. "When you have an exacerbation of this, it is a disgusting disease you know, and you have a poor selfimage" (male, 18-64 yrs).

Socially, patients' home and working lives were affected by the bronchiectasis; one patient couldn't cope after having a bad exacerbation and had to live with her daughter for a period of time; another patient had to regularly take time of work. "I seem to be having an awful lot of time off work...you can't guarantee that one week you're OK, and the next week you are maybe off again" (female, 18-64 yrs).

\section{Core category 2: self-management strategies}

Some patients with bronchiectasis in this study perceived selfmanagement as an approach independent from the health professional and others perceived self-management as a treatment regimen directed by a health professional, as illustrated by the following quotes. "To me self-management began when I was first diagnosed. First of all finding out as much as I could about the condition, what it is, what causes, what possible causes of it. There are a number of causes of it some of them unknown. What sort of treatments, in terms of medication and self-help should I be looking to, and to do that I hit the Internet" (male, 18-64 yrs); and "Self-management to me means when I get up in the mornings I have to do postural drainage, and sort of clapping of the lungs" (female, 18-64 yrs).

Patients with bronchiectasis perceived they were engaging in a range of self-management strategies (table 4).

Subcategories consistent across all four focus groups included antibiotic therapy, performing airway clearance, having an exercise regimen, complementary and alternative medicine and problem solving as illustrated by the following quotes. "I always think it is a good thing to have antibiotics in the house" (female, $\geqslant 65 \mathrm{yrs}$ ); "I do postural drainage every morning" (female, 18-64 yrs); "Exercise is the biggest component of my self help there is no doubt about that" (male, 18-64 yrs); "I take a cod liver oil capsule every morning" (male, $\geqslant 65 \mathrm{yrs}$ ); and "I'd a problem with the garden, lawn mowing. I've got a large garden but I bought a ride-on lawn mower" (male 18-64 yrs).

Most patients were prescribed inhaled or nebulised therapy and the majority of patients agreed that it was important they received annual influenza vaccinations, with some having also received the pneumococcal vaccination. Nutritional advice appeared to be lacking in all four focus groups. Monitoring of bronchiectasis was evident by the patient's ability to differentiate symptoms between stable and exacerbation phases, but patients reported a wide variation in symptoms during an exacerbation. A small number of patients made a decision to avoid situations or stimulants that would irritate their bronchiectasis, as part of their self-management approach: "I went to the pub quite a lot but now I don't. About once a week I would go because I just can't stick the smoke in the place" (male, 18-64 yrs).

Some patients had built up relationships with healthcare professionals including their general practitioner (GP) and

\section{TABLE 3 Core category: physical and psychosocial impact of bronchiectasis}

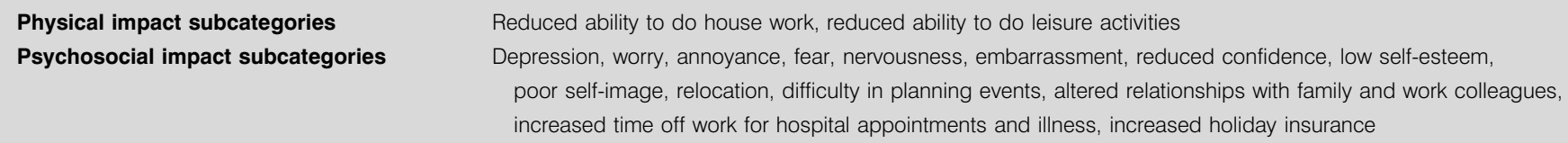




\section{TABLE 4 Core category: self-management strategies currently used by patients with bronchiectasis \\ Adherence to medical treatments subcategories \\ Prevention of ill health/promotion of good health subcategories \\ Dealing with psychological and social needs subcategories \\ Communication subcategories \\ Self-administration of intravenous antibiotics, having reserve oral antibiotics, performing airway clearance, using inhaled and nebulised therapies, receiving vaccinations Monitoring of symptoms and signs, sending a sputum sample for testing, exercising, dietary changes, reducing alcohol intake, smoking cessation, avoidance of irritants, complementary and alternative medicine \\ Attending a psychiatrist, acceptance, problem solving, attending an evening class, employing a housekeeper \\ Contacting the bronchiectasis multidisciplinary team, general practitioner, pharmacist, social services, ambulance service, insurance company}

pharmacist. Having a named contact person at the bronchiectasis service was seen as useful.

\section{Core category 3: obstacles to self-management}

Patients with bronchiectasis perceived a number of obstacles to self-management; the subcategories identified related to bronchiectasis, other health issues, and psychological and social obstacles (table 5).

The most consistent obstacle to self-management, discussed in all four focus groups, was in relation to disease severity/ stability. Patients did not initiate or continue self-management strategies if they had no symptoms or little change in their symptoms: "I have been with the physios twice, but I have no regime of management...because I don't believe I've got a severe case of this (bronchiectasis)" (male, 18-64 yrs).

Patients perceived that hospital and primary-care practices caused a delay in medical treatment. Some patients felt there was an unnecessary time lag between sending a sputum sample for testing and obtaining the results. Patients had difficulty getting reserve antibiotics, especially when going on holiday and others didn't like taking "a lot of" antibiotics as they experienced thrush as a side-effect. Patients tended to be aware of the symptoms of bronchiectasis but some were unaware of what bronchiectasis was, its prognosis, how it affected the lungs and the significance of test results: "The only thing ever I was told was, that's very good (spirometry). I don't know what very good would be...I always come away a wee bit frustrated" (male, $\geqslant 65$ yrs).

Psychological influences tended to prevent patients from engaging in self-management or limited the effectiveness of self-management: "Motivation's hard for me you know to get back to swimming" (male, 18-64 yrs); and "I say to myself when I'm doing it (postural drainage)...I wonder is this all useless, am I doing it right" (female, $\geqslant 65 \mathrm{yrs}$ ).

Social obstacles to self-management influenced healthcare regimes; for example, patients stopped their medical treatment in favour of holidays, or due to lack of time: "I was going away...for a week so I had some wine every night...it just happened to be when I was on this six weeks course (of antibiotics), so I fell by the wayside" (male, $\geqslant 65 \mathrm{yrs}$ ).

\section{Core category 4: sources of support for self-management}

Patients with bronchiectasis identified sources of support that would encourage them to self-manage (table 6).

The most consistent sources of support for self-management, discussed in all four focus groups, were information and guidance on the disease and accessibility to healthcare. Patients were keen to have a greater knowledge about bronchiectasis and more open access to healthcare services as illustrated by the following quotations: "If I understood more what to do you know I could try and help it myself. I don't want to come in to hospital" (male, 18-64 yrs); and "If there was some sort of support for a bronchiectasis patient who knows that they're running into trouble...there just seems to be delays all the time in trying to get you what you need at the time" (female, 18-64 yrs).

Some patients indicated that they would like to have reviews to ensure they were using self-management strategies correctly, and some patients indicated that they were more inclined to engage in self-management strategies if the benefits of treatment were evident. Close proximity to leisure facilities was identified as a factor that would encourage patients to engage in exercise as a self-management strategy. The government initiative for a smoking ban in public houses was also seen as a relevant factor in self-management.

The role of family was identified as important in self-management, particularly in giving advice, providing support and assisting with treatment of the patient with bronchiectasis, especially airway clearance. The patient's position within the family, such as a single parent, increased willingness to participate in self-management to avoid admission to hospital. "It's one of the reasons why I'm out every day cycling, why I force it because I have to just keep out of hospital...it keeps me focused" (male, 18-64 yrs).

TABLE 5 Core category: perceived obstacles to self-management of bronchiectasis

Disease-related obstacles subcategories

Psychological obstacles subcategories

Social obstacles subcategories
Disease severity/stability, health service policies, lack of information on disease process, side-effects of treatment, complications of bronchiectasis, other medical conditions, advancing age

Fear, lack of confidence, lack of competence, lack of motivation, lack of support

Personal circumstances, lifestyle preferences, financial reasons, lack of time, adverse weather conditions 


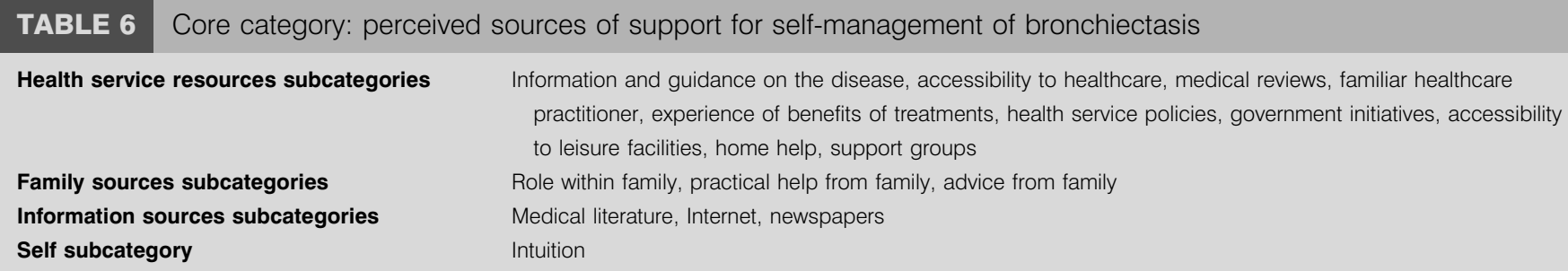

Health service resources subcategories

Family sources subcategories

Information sources subcategories

Self subcategory

Information and guidance on the disease, accessibility to healthcare, medical reviews, familiar healthcare practitioner, experience of benefits of treatments, health service policies, government initiatives, accessibility to leisure facilities, home help, support groups

Role within family, practical help from family, advice from family

Medical literature, Internet, newspapers

Intuition

Patients mentioned seeking information on bronchiectasis in medical books and on the Internet, but it was observed that patients generally relied on health professionals to provide relevant educational material. Self-awareness or intuition was seen as an inbuilt source of support for self-management. "I think by the time somebody would say to you you're not well you would know yourself" (female, $\geqslant 65 \mathrm{yrs}$ ).

\section{DISCUSSION}

The present study showed that patients with bronchiectasis have their lives disrupted by this long-term condition and can utilise self-management strategies. There are perceived obstacles to self-management but there are also perceived sources of support for self-management.

The emphasis on self-management of long-term conditions is growing and is seen as essential to enhance care in the Chronic Care Model endorsed by the WHO [5]. This model suggests that patients' functional and clinical outcomes can be improved by informed and activated patients who selfmanage, and by proactive healthcare providers. Access to necessary healthcare, self-management and decision support, and use of clinical information systems is central to the Chronic Care Model.

In the present study, patients had conflicting views on the meaning of self-management but self-management strategies generally involved using and adapting medical treatments that had been prescribed by health professionals. Patients appeared to monitor their condition and initiate or alter their treatment regimens during an exacerbation, for example, starting reserve antibiotics. They appeared to have no real problems communicating with health professionals when necessary, but lacked the skills to self-manage psychological and social needs. The majority of patients appeared to be comfortable with the opportunity to self-manage, especially if it meant staying out of hospital, but some patients lacked confidence and felt they would like assistance from community healthcare resources, or to be admitted to hospital if they were ill.

Existing research on self-management in respiratory conditions has been focused on asthma, COPD and CF and has examined the motivation for patients to self-manage, the influence of education programmes on self-management, the effectiveness of action plans to guide self-management, methods to control self-management of symptoms, and the impact of social factors on self-management [14-18]. There are no known studies on self-management in bronchiectasis, but the efficacy of some of the interventions self-managed by the patients has been investigated. Oral antibiotic therapy is frequently prescribed to patients with bronchiectasis and found to be effective in eradication of purulent secretions in this patient population [19]. Self-administration of intravenous antibiotics is available for patients with bronchiectasis and it is reported that patients prefer this method to hospital treatment as it promotes independence [20]. Home intravenous antibiotics may, however, increase medication costs as patients may report more exacerbations than previously, when the only alternative was admission to hospital for treatment [21]. Inhaled therapy is frequently prescribed to patients with bronchiectasis and, in the present study, most of the patients were using bronchodilators on a regular basis [22]. It has been shown there is a significant response to bronchodilators in this patient population [23]. Patients with bronchiectasis often have an increase in sputum production and are advised to incorporate airway clearance techniques into their treatment regimen. Techniques involve breathing exercises (the active cycle of breathing techniques) or positive pressure adjuncts (positive expiratory pressure mask, flutter, Acapella ${ }^{\circledR}$ (DHD Healthcare, Aumsville, NY, USA)). The bulk of existing literature on airway clearance techniques in bronchiectasis compares the efficacy of one technique with another [24-26]. Reported clinical benefits of airway clearance include reduced cough, a decrease in shortness of breath, an improvement in exercise capacity and a better quality of life [27]. Exercise, another popular self-management strategy in the present study, is known to improve both physical and mental health in a wide range of chronic conditions, and in patients with bronchiectasis a pulmonary rehabilitation programme has been shown to improve aerobic capacity, endurance exercise capacity and also inspiratory muscle strength [28]. The use of complementary and alternative medicine (CAM) was a consistent self-management strategy used in all four focus groups with cod liver oil being the most frequently used product, followed by multivitamins, echinacea and evening primrose oil. Tai Chi was also practiced by one patient and acupuncture had been used by another patient. There are no studies investigating the efficacy of CAM in bronchiectasis, but studies in asthma and COPD have highlighted mixed results for effectiveness and safety [29].

Obstacles to self-management highlighted some sex and age differences, although these were minimal between groups. One of the mothers in a female focus group highlighted that a lack of time for airway clearance was one of her main obstacles for this self-management strategy. Motivation was identified as an obstacle to exercise in both younger groups, especially in relation to swimming. Both younger groups (18-64 yrs) also discussed side-effects of treatment as obstacles to their selfmanagement strategies; one male subject did not like taking antibiotics as he suffered from thrush as a result, and one female subject only used her inhalers if she really needed them, 
as she had an irregular heart beat and perceived that the inhaler could "kick that off". In the two older groups $(\geqslant 65 \mathrm{yrs})$, age and lack of confidence were seen as obstacles to self-management. Age was seen as another barrier to exercise and lack of confidence made one female subject worry whether she was doing her airway clearance technique correctly and deterred one male subject from self-administering his intravenous antibiotics. If the perceived obstacles to self-management were addressed through education, with guidance and support from healthcare professionals then the majority of patients with bronchiectasis could be encouraged to be more independent with many aspects of their care.

Patient perceptions in this study indicate that important elements to support self-management not only include health professionals, but also themselves and their families. The role of the healthcare professional is seen as important for information and guidance on the condition and review of selfmanagement strategies. Patients would also like to have access to services in times of need.

The results of the current study support a number of current models of health related behaviour. The models explain why some patients may self-manage better than others. The social learning theory considers a person's health-related behaviour to be related to expectancy and value of a certain outcome [30]. Examples of this in the current study were seen when patients stated that they saw the advantage of adhering to medical treatments such as inhaled therapy or airway clearance to maintain status quo. Self-efficacy is important in Bandura's theory of social learning, which relates to patients believing they are competent to perform a specific task [30]. One example of self-efficacy in the present study was when patients reported that they felt competent to self-administer home intravenous antibiotic therapy. Fishbein's theory of reasoned action considers health-related behaviour to be influenced by the individual's attitude and social expectations [30]. Examples of this were seen when patients stated that they followed the advice given to them by family members because they believed it to be important.

The health belief model considers cues to action, whether external or internal, will predict health-related behaviour [30]. Examples of this model were seen when patients stated that they followed the advice given in medical literature (external cue), or monitored their symptoms (internal cue) and sought help when an exacerbation occurred. Finally, attribution theory considers past outcomes to the patient or other patients as relevant in health-related behaviour [30]. It suggests that feeling in control is important, for example when the current study patients stated that they avoided known irritants that aggravated their bronchiectasis.

There is no single theory that explains all health-related behaviour of patients with bronchiectasis and the results from this study support several concepts. When developing a selfmanagement programme, it is important to consider theories of health-related behaviour as they may predict variation in outcome. It is also important to consider that self-management programmes may not be equally effective in all respiratory conditions and assessment of the efficacy of self-management in bronchiectasis needs to be established [31-33].
There are some limitations to the present study. Focus groups, by their nature, are qualitative and thus the results are difficult to quantify. The majority of patients at the focus groups lived in a city and thus were in close proximity to their GP and hospital services. Patients living in rural areas may have different experiences, for example, in obtaining antibiotics. Patients who were more severely ill and on oxygen therapy may also have had different experiences of the bronchiectasis and also different needs.

\section{Conclusion}

In summary, the present study has given a unique insight into the experience of patients with bronchiectasis, whose lives are disrupted by this chronic condition. The findings indicate that patients are utilising self-management strategies. There are a number of perceived obstacles to self-management but there are also perceived sources of support for self-management. The study has provided information, from the patients' perspective, of elements that need to be included in a disease-specific self-management programme.

\section{REFERENCES}

1 Wilson CB, Jones PW, O'Leary CJ, et al. Systemic markers of inflammation in stable bronchiectasis. Eur Respir J 1998; 12: 820-824.

2 Lavery K, Bradley JM, Elborn JS. Bronchiectasis: challenges in diagnosis and management. Int J Respir Care 2005; 1: 92-98.

3 Martinez-Garcia MA, Perpina-Tordera M, RomanSanchez P, Soler-Cataluna JJ. Quality of life determinants in patients with clinically stable bronchiectasis. Chest 2005; 128: 739-745.

4 O'Leary CJ, Wilson CB, Hansell DM, Cole PJ, Wilson R, Jones PW. Relationship between psychological well-being and lung health status in patients with bronchiectasis. Respir Med 2002; 96: 686-692.

5 Epping-Jordan JE, Pruitt SD, Bengoa R, Wagner EH. Improving the quality of health care for chronic conditions. Qual Saf Health Care 2004; 13: 299-305.

6 Department of Health. Self care - A real choice: Self care support - A practical option, 2005. www.dh.gov.uk/ assetRoot/04/10/17/02/04101702.pdf. Date last accessed: April 19, 2006. Date last updated: January 12, 2005.

7 Long-term Medical Conditions Alliance. Supporting Expert Patients Resource Pack, 2001. www.lmca.org.uk/ pdfs/SM\%20folder.pdf. Date last accessed: April 19, 2006.

8 Gibson PG, Powell H, Coughlan J, et al. Self-management education and regular practitioner review for adults with asthma. Cochrane Database Syst Rev 2002; 3: CD001117.

9 Monninkhof EM, van der Valk PDLPM, van der Palin J, et al. Self-management education for chronic obstructive pulmonary disease. Cochrane Database Syst Rev 2002; 4: CD002990.

10 Bartholomew LK, Czyzewski DI, Parcel GS, et al. Selfmanagement of cystic fibrosis: short-term outcomes of the Cystic Fibrosis Family Education Program. Health Educ Behav 1997; 24: 652-666.

11 Wouters EFM. Blending pulmonary rehabilitation into the WHO view. Int J Respir Care 2005; 1: 77. 
12 Burrows D, Kendall S. Focus groups: what are they and how can they be used in nursing and health care research? Social Sciences in Health 1997; 4: 244-253.

13 Global Initiative for Chronic Obstructive Lung Disease. Executive Summary, Sept 2005. www.goldcopd.com/ Guidelinesresources.asp $|1=2 \&| 2=0$. Date last accessed: April 19, 2006. Date last updated: November, 2006.

14 Steven K, Morrison J, Drummond N. Lay versus professional motivation for asthma treatment: a cross-sectional, qualitative study in a single Glasgow general practice. Fam Prac 2002; 19: 172-177.

15 Parcel GS, Swank PR, Mariotto MJ, et al. Self-management of cystic fibrosis: a structural model for educational and behavioral variables. Social Science Med 1994; 38: 1307-1315.

16 Turner MO, Taylor D, Bennett R, Fitzgerald JM. A randomized trial comparing peak expiratory flow and symptom self-management plans for patients with asthma attending a primary care clinic. Am J Respir Crit Care Med 1998; 157: 540-546.

17 Nguyen HQ, Carrieri-Kohlman V. Dyspnea self-management in patients with chronic obstructive pulmonary disease: moderating effects of depressed mood. Psychosomatics 2005; 46: 402-410.

18 Osman L. Health habits and illness behaviour: social factors in patient self-management. Respir Med 1998; 92: 150-155.

19 Hill SL, Morrison HM, Burnett D, Stockley RA. Short term response of patients with bronchiectasis to treatment with amoxycillin given in standard or high doses orally or by inhalation. Thorax 1986; 41: 559-565.

20 Winter RJD, George RJD, Deacock SJ, Shee CD, Geddes DM. Self-administered home intravenous antibiotic therapy in bronchiectasis and adult cystic fibrosis. Lancet 1984; 16: 1338-1339.

21 Conway A. Home intravenous therapy for bronchiectasis patients. Nursing Times 1996; 92: 34-35.

22 O'Neill B, Bradley JM, MacMahon J, Elborn JS. Subjective benefit of inhaled therapies in patients with bronchiectasis: a questionnaire study. Int J Clin Practice 2004; 58: 441-443.
23 Hassan JA, Saadiah S, Roslan H, Zainudin BMZ. Bronchodilator response to inhaled beta-2 agonist and anticholinergic drugs in patients with bronchiectasis. Respirology 1999; 4: 423-426.

24 Patterson JE, Bradley JM, Elborn JS. Airway clearance in bronchiectasis: a randomized crossover trial of active cycle of breathing techniques (incorporating postural drainage and vibration) versus test of incremental respiratory endurance. Chron Respir Dis 2004; 1: 127-130.

25 Thompson CS, Harrison S, Ashley J, Day K, Smith DL. Randomised crossover study of the Flutter device and the active cycle of breathing technique in non-cystic fibrosis bronchiectasis. Thorax 2002; 57: 446-448.

26 Patterson JE, Bradley JM, Hewitt O, Bradbury I, Elborn JS. Airway clearance in bronchiectasis: a randomized crossover trial of active cycle of breathing techniques (ACBT), versus Acapella®. Respiration 2005; 72: 239-242.

27 Pryor JA. Physical therapy for adults with bronchiectasis. Clin Pulm Med 2004; 11: 201-209.

28 Newall C, Stockley RA, Hill SL. Exercise training and inspiratory muscle training in patients with bronchiectasis. Thorax 2005; 60: 943-948.

29 Lewith GT. Respiratory illness: a complementary perspective. Thorax 1998; 53: 898-904.

30 Marteau T. Health beliefs and attributions. In: Broome A, Llewelyn S., eds. Health Psychology, Process and Applications. 2nd Edn. London, Chapman \& Hall, 1995; pp. 3-20.

31 Jones A, Pill R, Adams S. Qualitative study of views of health professionals and patients on guided self-management plans for asthma. BMJ 2000; 321: 1507-1510.

32 Monninkhof E, van der Valk P, Schermer T, van der Paten J, van Herwaarden C, Zielhuis G. Economic evaluation of a comprehensive self-management programme in patients with moderate to severe chronic obstructive pulmonary disease. Chron Respir Dis 2004; 1: 7-16.

33 Bourbeau J. Not all self-management programs in chronic obstructive pulmonary disease have positive results: why is replication a problem? Chron Respir Dis 2004; 1: 5-6. 